\section{Global WHO survey: poor physical and mental health more prevalent among women who have experienced intimate partner violence}

\section{QUESTION}

Question: Is intimate partner violence and domestic violence linked to physical and mental health problems in women?

Population: 19568 inhabitants of 10 countries who had ever had a partner.

Setting: Global study representing people from 10 countries (15 sites) in the WHO multi-country study on women's health and domestic violence against women; interviews carried out 2000-2003.

Assessment: Interviews were conducted in person by female interviewers in a community setting using a standardised questionnaire. Women were asked whether they had experienced physical or sexual violence at the hand of a current or previous partner at any time in their life or in the previous 12 months. Women responding yes were then asked for details of the incidents. Other questions included selfrated health and whether they experienced any of the following physical symptoms in the previous 4 weeks: difficulty walking or with daily activities, pain, memory loss, dizziness and vaginal discharge. The Self Report Questionnaire (SRO-20) was used to screen for emotional distress (asking about symptoms such as crying, tiredness and inability to enjoy life), and participants were also asked whether they had suicidal thoughts in the 4 weeks prior to interview or had ever attempted to commit suicide. Results were pooled using multiple regression models. Analysis was adjusted for age, gender, site, education and marital status.

Outcomes: Physical and sexual abuse; self-reported health and specific physical health problems; emotional distress, suicidal thoughts and suicide attempts; injury from violence.

\section{METHODS}

Design: Cross sectional study.

\section{MAIN RESULTS}

Lifetime prevalence of physical or sexual violence by a current or former partner among ever-partnered women ranged from $15 \%$ in Japan to $71 \%$ in Ethiopia. Among women who had been physically abused the prevalence of injury from violence ranged from 19\% in Ethiopia to 55\% in Peru. Overall, women with a lifetime experience of physical or sexual violence were more likely to report poor or very poor health (OR 1.6, 95\% CI 1.5 to 1.8$)$. They were also more likely to report difficulties walking (OR 1.6, 95\% CI 1.5 to 1.7) and with their daily activities (OR 1.6, 95\% CI 1.5 to 1.8), pain (OR 1.6, 95\% CI 1.5 to 1.7 ), memory loss (OR $1.8,95 \%$ CI 1.6 to 2.0 ), dizziness (OR 1.7, 95\% CI 1.6 to 1.8 ) and vaginal discharge (OR 1.8, 95\% CI 1.7 to 2.0). Across all sites, emotional distress on the SRO-20 was significantly higher for women who had experienced abuse as was the risk of suicide ideation (OR $2.9,95 \%$ CI 2.7 to 3.2 ) and suicide attempts (OR 3.8, 95\% CI 3.3 to 4.5$)$.

\section{CONCLUSIONS}

There are associations between lifetime experiences of intimate partner violence and poor physical and mental health in women.

\section{ABSTRACTED FROM}

Ellsberg M, Jansen $\mathrm{H}$, Heise L, et al. Intimate partner violence and women's physical and mental health in the WHO multicountry study on women's health and domestic violence: an observational study. Lancet 2008;371:1165-72.

Correspondence to: Dr C Garcia-Moreno, Department of Reproductive Health and Research, WHO, 20 Avenue Appia, 1211 Geneva 27-CH, Switzerland; garciamorenoc@who.int

Source of funding: World Health Organisation; Governments of the Netherlands, Norway, Sweden, Switzerland, and UK; Rockefeller Foundation; Urban Primary Health Care Project of the Government of Bangladesh; Swedish Agency for Research Cooperation with Developing Countries (SAREC/Sida); United Nations Fund for Population Activities (UNFPA); and Trocaire.

- Additional notes are published online only at http://ebmh.bmj.com/content/vol11/ issue 4
E Ilsberg and colleagues provide compelling data characterising the association between disease burden and intimate partner violence (IPV)—a prevalent but under-recognised human rights violation. The study included a large sample of women from 10 countries and found a high prevalence of IPV $(15 \%-71 \%$ of women reported lifetime physical and/or sexual IPV victimisation), and strong associations between IPV victimisation and injury, emotional distress, suicidal ideation, suicide attempts, functional limitations and poor health.

Despite some limitations in the design (eg, crosssectional) and measurement (eg, physical and sexual violence were pooled as a single exposure), the study elucidates a crucial finding that irrespective of cultural and social context, IPV victimisation in women presents a significant disease burden extending beyond physical injury. In spite of this, prevention efforts are hampered by gender inequities, poverty, societal structures that support violence against women and a lack of funding.

Prevention efforts are needed that address gender inequities and structures that constrain women's rights, improve services for women victimised by violence, and ensure adequate allocation of dollars to support organisations and research addressing violence against women. The reality of limited infrastructure in middle and low resource countries calls for innovation and coordinated participation from key stakeholders.

Promising global violence prevention strategies include: (1) targeting families in which women have little decision-making power and changing attitudes and behaviours that perpetuate violence, such as the Raising Voices program in Uganda and Tanzania; and (2) linking income generating programmes to curricula on gender inequity, reproductive health and HIV/AIDS prevention, like the IMAGE programme in South Africa. Emphasising men's role in ending violence against women and intervening with youth are also priorities; a school-based programme reduced teen dating violence up to 4 years after implementation. ${ }^{1}$ Without urgent prevention efforts, the violence pandemic will continue.

\section{Amy E Bonomi, PhD, MPH}

Human Development and Family Science, The Ohio State University, Columbus, Ohio, USA

\section{Nancy Glass, PhD, MPH, RN}

Center for Global Health, Johns Hopkins University, Baltimore, Maryland, USA

Competing interests: None.

1. Foshee VA, Bauman KE, Ennett ST, et al. Assessing the long-term effects of the Safe Dates program and a booster in preventing and reducing adolescent dating violence victimization and perpetration. Am J Public Health 2004:94:619-24. 